\title{
Marcel Fournier, Charles Kraemer (dir.), Durkheim avant Durkheim. Une jeunesse vosgienne
}

Paris, L'Harmattan, coll. « Socio-anthropologie », 2014, 266 p.

\section{Matthieu Béra}

\section{(2) OpenEdition}

\section{Journals}

Édition électronique

URL : http://journals.openedition.org/assr/27360

DOI : $10.4000 /$ assr. 27360

ISSN : $1777-5825$

Éditeur

Éditions de l'EHESS

Édition imprimée

Date de publication : 1 octobre 2015

Pagination : 298

ISBN : 978-2-7132-2515-4

ISSN : 0335-5985

\section{Référence électronique}

Matthieu Béra, « Marcel Fournier, Charles Kraemer (dir.), Durkheim avant Durkheim. Une jeunesse vosgienne », Archives de sciences sociales des religions [En ligne], 172 I octobre-décembre, mis en ligne le 20 mai 2016, consulté le 24 septembre 2020. URL : http://journals.openedition.org/assr/27360 ;

DOI : https://doi.org/10.4000/assr.27360

Ce document a été généré automatiquement le 24 septembre 2020

(C) Archives de sciences sociales des religions 


\section{Marcel Fournier, Charles Kraemer (dir.), Durkheim avant Durkheim. Une jeunesse vosgienne}

Paris, L'Harmattan, coll. « Socio-anthropologie », 2014, 266 p.

Matthieu Béra

\section{RÉFÉRENCE}

Marcel Fournier, Charles Kraemer (dir.), Durkheim avant Durkheim. Une jeunesse vosgienne, Paris, L'Harmattan, coll. « Socio-anthropologie », 2014, 266 p. 
1 Cet ouvrage restitue un grand nombre de recherches, souvent pointues, communiquées au public il y a maintenant sept ans (en mai 2008) à Épinal, à l'occasion des 150 ans de la naissance de Durkheim dans cette ville en 1858. La biographie d'Émile Durkheim par Marcel Fournier venait alors de paraître (Durkheim, 2007, Fayard); le projet de Fournier, co-organisateur des trois journées, était de reconstituer le cadre social de la "prime jeunesse» de Durkheim, de remonter aux sources, d'immerger les chercheurs et auditeurs dans son bain originel. Selon son biographe québécois, ce cadre séminal pourrait se caractériser par des éléments spirituel (le judaïsme), géographique (Épinal, les Vosges) et temporel (la guerre de 1870). Si Durkheim est devenu

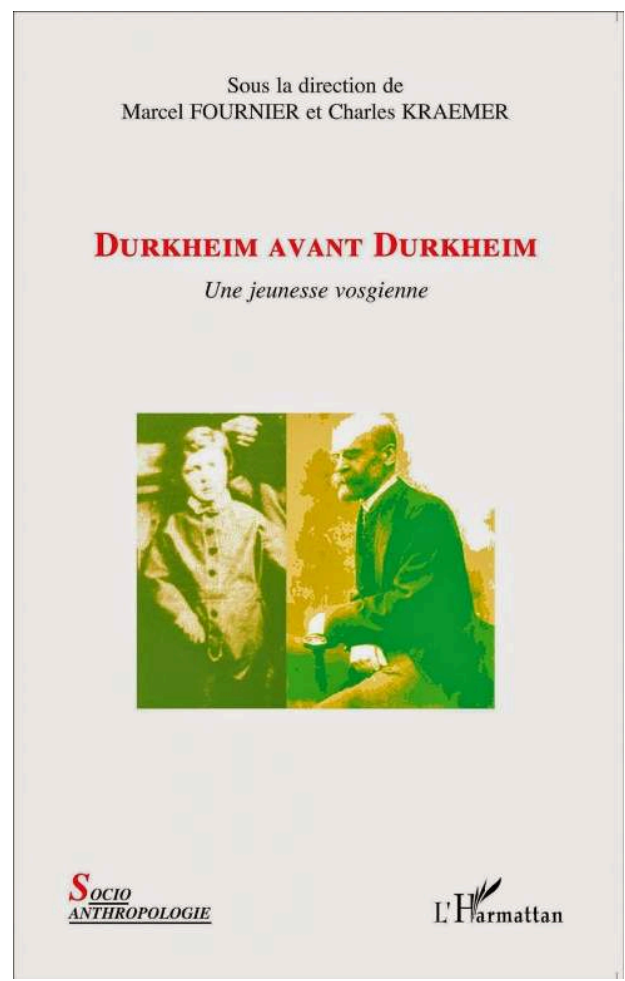
Durkheim (et si la sociologie française est ce qu'elle est), sans doute est-ce dû en partie à ces trois éléments - d'où l'intérêt de les connaître avec la plus grande justesse possible. Pour ce faire, il a fait appel à des historiens (les titres et les charges ne sont pas précisés, ce qui est un peu regrettable), spécialistes d'Épinal, des Vosges, de la guerre de 1870, du judaïsme, etc. L'ouvrage se décompose en trois parties: «la famille », « la communauté » [juive] et « la société ». Les intitulés de ce triptyque ne sont pas sans rappeler le classique Communauté et société (1885) de Tönnies, mais ce n'est qu'une évocation qui vient en toile de fond, un écho lointain non théorisé. Ici, la « famille » renvoie surtout aux ancêtres masculins (quand ils étaient rabbins) et au père de Durkheim. La « communauté » renvoie au judaïsme, tandis que la « société » renvoie à la guerre, au développement démographique et urbain et au cursus dans le secondaire.

2 Sur la famille, on trouvera quatre articles, dont celui de Pascal Faustini, bibliothécaire, qui reconstitue les origines rabbiniques des lointains ancêtres de Durkheim. On y trouve rassemblés les éléments concrets auxquels se réfèrent ceux qui écrivent (souvent sans savoir) que Durkheim « descend d'une famille de rabbins depuis plusieurs générations ». Cette fois, on sait précisément de quel côté (paternel), dans quelle région et dans quelle mesure "Durkheim porte en lui toute l'histoire du judaïsme rhénan depuis le Moyen Âge, une histoire faite d'expulsions et de migrations plus subies que choisies » (p. 20).

3 Un second article, de Simon Schwarzfuchs porte sur le rabbin Moïse (1805-1896), le père de Durkheim, lui-même fils d'Abraham Israël, instituteur à Haguenau et rabbin de Mutzig (il succéda à son beau-père à ce poste). Inutile de préciser que ce détour par celui qu'on pourrait surnommer le «grand-père (biologique) de la sociologie française " a une très grande valeur, d'autant que son influence (indéterminée en l'état actuel des connaissances) a pu être considérable sur son fils cadet resté à Épinal jusque 
18 ans, avant de partir étudier la philosophie à Paris, projet qui semble avoir animé le jeune Moïse autour de 1830 ( $c f$. p. 28). Son parcours est reconstitué, autant que faire se peut. Il suivit notamment une formation dans une Yeshiva (à ne pas confondre avec une école rabbinique en cela qu'on n'y délivre aucun diplôme). On apprend ses difficultés pour obtenir les certificats nécessaires à sa demande de poste: depuis 1831, l'État s'engageait à payer les rabbins de France en échange de quelques garanties. Or Moïse Durkheim n'avait ni le Bac, ni de certificat de langue française (il était né en Allemagne), ni de publication à faire prévaloir. «Il fut donc condamné à une carrière assez médiocre, sans véritable espoir d'avancement » (p. 38), commençant à $600 \mathrm{~F} / \mathrm{an}$ (et non par mois comme il est indiqué par erreur p. 17) en 1837, au point que l'auteur se demande : «Moïse a-t-il regretté d'avoir embrassé la profession rabbinique?». La situation financière du rabbin a pu inciter Durkheim à préparer l'École normale supérieure et le père à encourager son fils. Il faut se rendre compte que la seule réussite à ce concours permit à Durkheim de toucher d'emblée le double du traitement de son père (environ $1500 \mathrm{~F} / \mathrm{an}$ pour un boursier)! Le texte présenté, malheureusement, ne reprend pas les passages de la communication de S. Schwarzfuchs quand il évoqua le contenu des études de Moïse à Francfort auprès du rabbin Trier. Il avait pu les reconstituer de manière indirecte en retrouvant les écrits autobiographiques d'un autre élève qui avait été son contemporain, Alexandre Weil. Quoi qu'il en soit, il reste encore beaucoup de pistes à éclairer du côté du père et, partant, de l'éducation reçue par Émile Durkheim qui, ne l'oublions pas, devint un spécialiste de l'éducation. Sans être passé par une école rabbinique ni par une Yeshiva, il a malgré tout "subi" l'influence de son père ; comme il l'écrit en 1894 dans les Règles de la méthode sociologique, usant d'un je générique : «Je n'ai pas voulu l'éducation que j'ai reçue ; or, c'est elle qui, plus que toute autre cause, me fixe au sol natal ». De quelle nature a-t-elle pu être ? La question reste ouverte.

4 Vient ensuite l'article de Fournier qui met en parallèle les trajectoires de Durkheim et de Mauss. Ce lien familial est bien connu entre l'oncle maternel et son neveu plus jeune de 14 ans (ils sont nés respectivement en 1858 et 1872); ils passèrent par le même collège et vécurent leur enfance dans la même ville et la même famille. Fournier en profite pour évoquer la question religieuse (les fêtes) et les difficultés qu'avait Durkheim à se plier aux rites alimentaires lors de ses séjours à Épinal à la période de Pâques.

On peut regretter qu'il manque quelques contributions entendues à Épinal : notamment celle qui porta sur la mère de Durkheim, Mélanie Isodor Marx. On se doute pourtant qu'elle fut aussi un personnage central, si l'on veut se pencher sur son enfance et sa famille. Les journées de mai 2008 avaient permis d'approcher d'un peu plus près cette fille de vendeur aisé de chevaux qui devint gérante d'une petite entreprise de main d'œuvre textile ( $c f$. la communication de Claire Prévost, « Le travail à domicile au temps de Mélanie »). Elle avait en effet monté cette affaire, qui fut reprise par son fils aîné (Félix) puis par sa fille Rosine (mère de Mauss) pour améliorer la situation financière de la famille trop fragilisée par le seul traitement du père rabbin. Notons au passage que rien n'a été proposé sur les destins des frères et sœurs de Durkheim. Les fratries sont pourtant aussi intéressantes que les ascendants. On sait que Durkheim fut le dernier de quatre. Son grand frère Félix, décédé assez mystérieusement autour de 30 ans en 1887, est absent des biographies. Il aurait été intéressant de comprendre pourquoi cet aîné, pas plus que son petit frère Émile, ne fut orienté vers le rabbinat, mais plutôt vers le négoce de tissu, comme sa mère et sa sœur Rosine. Celle-ci, mère de Mauss et grande 
sœur d'Émile, est restée en relation épistolaire toute sa vie avec son fils aîné et son frère cadet. Sa correspondance est toujours inédite, elle est conservée par un descendant, en attente d'être publiée. Quant à Céline, la seconde sœur, elle eut aussi une descendance dont on ne sait encore rien.

Ainsi, cette première partie sur la famille porte déjà, on le voit, sur des questions qui touchent le judaïsme, de très loin le thème dominant de l'ouvrage, à tel point que son sous-titre "Une jeunesse vosgienne» nous semble un peu trompeur. «Une enfance juive " aurait été plus adéquate. À cet égard, l'ouvrage se place dans la lignée des travaux de Jean-Claude Filloux, bien étrangement effacé des références, qui datent des années 1970. Même remarque concernant les travaux de Pierre Birnbaum, notamment sa Géographie de l'espoir (Gallimard, 2004) qui consacre un chapitre entier à Durkheim et sa relation au judaïsme. Au final, rien n'est dit sur l'enseignement hébraïque qu'il put recevoir. On ne sait toujours pas s'il eût une éducation religieuse exclusive avant la $6^{\mathrm{e}} \mathrm{ni}$ dans quelle mesure il maîtrisait l'hébreu. À supposer qu'il reçut cette éducation religieuse, on ne sait pas en quoi elle consistait exactement. Qu'enseigna Moïse Durkheim à ses quatre enfants? On ne saurait donc dire jusqu'à quel point Durkheim rompit avec une éducation religieuse aux contours encore indistincts ni en quoi la sociologie durkheimienne fut inspirée par ce judaïsme. J.-C. Filloux tenta de répondre directement à cette question (et $\mathrm{P}$. Birnbaum aussi, bien que de manière plus externe). C'est vraisemblablement l'une des questions les plus intéressantes que l'on puisse se poser aujourd'hui, qui renvoie au lien entre l'enfance religieuse (et juive) de Durkheim et la sociologie française. Il reste à l'étudier de plus près, en proposant une analyse comparée des contenus afin de mettre à jour les homologies entre un enseignement religieux et ce que Durkheim théorisa ensuite. Le passage par le « collège » où il suivit des études classiques entre 1870 et 1876 , les trois années de "rhétorique supérieure " pour préparer l'ENS (1876 à 1879), l'enseignement à l'ENS (1879 à 1882), seront sans doute parvenus à supplanter une bonne partie de sa vision originelle du monde, du moins à la " transformer ». Filloux émit des propositions importantes à ce sujet dans les années 1970, il faudra y revenir (on pense notamment à la réticence durkheimienne à traiter de la question du conflit). On ne peut d'une main parier sur les origines et la socialisation intellectuelle et religieuse d'un homme et ne pas donner les éléments pour caractériser cette culture - un peu à la manière de ce que tenta Weber à l'échelle collective dans L'Éthique protestante et l'esprit du capitalisme, comparant "l'éthique protestante » à " l'esprit capitaliste », préalablement caractérisés en quelques traits essentiels.

7 Dans la seconde partie intitulée "La communauté » sont rassemblés les textes de Freddy Raphaël (le traducteur du Judaïsme antique de Weber en 1970), de Charles Kreamer (archéologue) et Alexandre Laumond (historien) et du même avec Gilles Grivel (historien). À eux seuls, ces deux derniers articles occupent une centaine de pages sur les deux cent cinquante que comporte l'ouvrage et les douze articles qui le composent. Ils s'intéressent à la communauté religieuse et proposent différents points de vue pour décrire la communauté juive des Vosges d'abord puis d'Épinal à la fin du XIXe. Nous passerons rapidement sur l'article de Raphaël «L'esprit du judaïsme d'Alsace », trop éloigné de Durkheim auquel il ne fait quasiment aucune allusion. C'est à notre avis un peu le travers de certaines contributions qui décrivent un contexte sans le relier au sujet du livre. 
8 Dans l'article de Laumond et Kreamer (co-directeur de cette publication), on découvre une carte de l'évolution de l'implantation des individus de confession juive dans les Vosges (p. 105). Cette technique rappelle la micro storia, dans la mesure où les ménages de cette communauté se comptent sur les doigts des deux mains : rarement plus d'une dizaine dans les villes des Vosges. Les registres d'état civil renseignent sur les confessions (ce fut le cas jusqu'en 1872) et fournissent des « indices » qui donnent une idée de la volonté d'intégration des communautés religieuses au creuset républicain. L'étude des prénoms (p.108) est un moyen de «mesurer» cette intégration à la « communauté nationale ». Entre 1830 et 1860, les choses évoluent effectivement, « la moitié des prénoms sont identiques à ceux donnés par les autres communautés » (p. 112). Les «prénoms identitaires» représentaient $90 \%$ du total en 1808 (p. 108). La répartition professionnelle des ménages permet également de caractériser la «communauté juive» et de comparer la situation de la famille Durkheim à cette norme. On apprend ainsi que les marchands de chevaux y sont très nombreux (ils représentent $27 \%$ du total des actifs recensés), comme le fut le grand-père maternel d'Émile. D'un autre côté, le métier de rabbin était très rare. Les individus recensés qui signalent leur confession juive ne sont à l'époque ni lettrés ni intellectuels. Dans les diagrammes de répartition de la page 115, on n'en compte aucun en 1875 à Épinal, et aucun en 1889 à Rambervilliers. Autant dire que la profession de Moïse, puis de son fils qui devint enseignant sont à la fois singulières et pionnières (c'est au lecteur de le déduire). De même, les deux auteurs de l'article étudient certains réseaux de sociabilités intellectuelle et associative: société philomathique, loges maçonniques, société d'émulation. Ils constatent que les Juifs y sont très peu présents en 1900 : moins de $5 \%$ sont repérés (par quel procédé ?) dans les listes - ce qui toutefois est plus que proportionnel à leur poids démographique (ce que semblent perdre de vue les auteurs quand ils s'étonnent de ce chiffre, $c f$. p. 134).

9 Après avoir étudié les Vosges, le troisième article se focalise sur Épinal. Le microscope se fait plus précis et on comprend l'intérêt de la méthode. On découvre qu'il n'y avait que dix-neuf Juifs à Épinal en 1806 (p. 149) dont trois arpenteurs géomètres (le détail est amusant quand on veut bien se souvenir de la profession du héros du Château de Kafka, « l'arpenteur K »). En 1813, ils sont passés à trente-sept. Quand Durkheim nait, on recense cent cinquante juifs. Ils seront même deux cent trente-six en 1869. Par conséquent, écrivent-ils, "Durkheim naît alors que la population juive spinalienne est en pleine croissance » (p. 154). Le fait est notable, certes, mais on ne voit pas très bien en quoi il pourrait avoir son importance pour Durkheim, son état d'esprit et sa psychologie. On aimerait connaître l'implicite des auteurs, s'il en est un. Une belle carte (p. 158) permet de localiser les logements des foyers de confession juive à Épinal. Les auteurs parviennent à saisir un phénomène très intéressant, l'endogamie confessionnelle, qui « est la règle absolue » : aucun mariage mixte n'est recensé ! C'est évidemment un autre indicateur de (non) intégration, peut-être plus pertinent que celui qui consiste à consulter les prénoms et les professions (il faudrait symétriquement le comparer à celui des protestants, des catholiques et des athées pour déterminer en quoi il serait propre ou non à une communauté religieuse). On sait que Durkheim luimême se mariera avec une jeune femme de confession juive (Julie Dreyfus) en 1887 et que leur fille Marie en fera de même avec Jacques Halphen en 1913. Plus que "d'intégration", cependant, il faudrait peut-être parler d'effacement progressif de l'imprégnation religieuse. C'est une chose que de "s'intégrer » en réalisant un mariage mixte au plan confessionnel, une autre que de renoncer à tout mariage confessionnel. 
C'est pour cela que les auteurs évoquent «la lente sécularisation de la communauté juive d'Épinal» (p.164) de 1890 à 1930, mesurée par l'intégration croissante de personnalités de confession juive parmi les élites administratives (un inspecteur des finances, un Directeur général au ministère des Finances), politiques (un vice-président du parti radical) et professionnelles (des médecins, architectes, avocats, enseignants). On le note aussi avec l'apparition des mariages mixtes et de l'exogamie. Sur cent quatorze couples recensés en 1936, 13 \% étaient exogames (p. 172).

La «société", troisième volet du triptyque renvoie à la guerre de 1870, à l'enseignement public, à la vie urbaine d'Épinal, à l'industrialisation et à la modernisation. Elle a eu aussi, on s'en doute, une influence sur Durkheim, peut-être plus importante que la famille et la communauté religieuse, plus ou moins conciliable avec l'éducation religieuse. De notre point de vue, c'est-à-dire 150 ans plus tard, on peut affirmer que le judaïsme des ancêtres n'a pas résisté aux effets de la vie urbaine, aux apprentissages scolaires, à la culture universitaire (littéraire, philosophique et scientifique), à l'éloignement familial et géographique. L'intégration républicaine de ce fils de la petite communauté juive d'Épinal ressemble à une assimilation en effaçant des pans entiers de l'éducation religieuse qu'il n'a pas transmise. Cependant, l'intégration dans la «République» est autant une intégration dans l'urbanisation, l'industrialisation, la modernité scientifique, des éléments qui ont peut-être moins à voir avec les valeurs de la République qu'on peut le lire parfois et plus à voir avec les effets du libéralisme et de la modernisation technique. Ces aspects (à peine) théoriques ne sont pas discutés dans l'ouvrage.

11 Cette partie se compose de trois articles. Le premier, très court (moins de huit pages dont quatre couvertes d'illustrations) de l'historien Jacques Grasser se propose de décrire "le milieu spinalien » dans lequel Durkheim vécut dix-huit ans, qu'il estime «sans doute déterminant» (p.198). Il se demande «quel a pu être l'apport [sic] du milieu social, géographique, historique spinalien de cette époque», pour ajouter aussitôt: "sans pouvoir répondre à cette question qui concerne précisément l'évolution de la personnalité de Durkheim, on peut, néanmoins, brièvement décrire quel fut cet environnement dans les années 1858-1876». Nous retrouvons cette limite de l'analyse « par le contexte » qui s'arrête où on attendrait qu'elle explicite la jointure, l'influence, que Grasser appelle "l'apport», sur l'individu. Que nous apprend la démographie? Il y avait 12000 habitants en 1866 à Épinal et une « révolution urbaine sociale, économique, presque en coïncidence avec la naissance de Durkheim». En liaison avec ce dynamisme démographique, on assiste à la naissance du tronçon de voie ferrée Nancy-Épinal en 1857. Plus déterminante que l'arrivée du train, il y eut la guerre de 1870. Durkheim « assiste à la courte bataille d'Épinal » et à l'occupation des troupes jusque juillet 1873, dans la ville qui se trouve à $45 \mathrm{~km}$ de la frontière avec la nouvelle Allemagne. La guerre engendre un afflux de populations nouvelles (15000 habitants en 1876, 26000 en 1891), des protestants alsaciens et quelques familles juives.

Plus intéressant (car plus développé) est l'article très documenté de l'historien Philippe Alexandre qui s'est informé sur tout ce qui avait trait à la vie du " collège » (entendre collège et lycée) où Durkheim fit de brillantes études secondaires. Cette fois, l'auteur a constamment rattaché son sujet (le collège) à Durkheim. On en ressort avec beaucoup d'informations inédites. Un tableau (p. 206) retrace la scolarité de l'enfant. On apprend aussi dans le rapport hebdomadaire du principal que Durkheim était passé de la $7^{\mathrm{e}}$ à la $5^{\mathrm{e}}$ et que l'inspecteur d'académie le félicita (p.237). Un autre tableau permet de 
connaître le nombre d'élèves par classe (p. 214). Ainsi, en $4^{\mathrm{e}}$, ils étaient dix. En philosophie (en 1870), ils étaient quatre! Il aurait été intéressant de retrouver le nombre d'élèves quand Durkheim était en terminale (en 1874/1875). Le tableau de la page 208 donne le nom de ses enseignants avec leur traitement en 1870. Ainsi, on apprend que le professeur de philosophie était l'abbé Marin, licencié ès lettres. En 1873, on apprend avec étonnement que le nouveau (?) professeur de philosophie se nomme « Fournier» (p. 221), lui aussi licencié ès lettres.

Le dernier article est de l'historien François Roth, spécialiste de la Lorraine et de la guerre de 1870. Il présente la guerre de 1870 au plus près d'Épinal et son impact sur l'enfant Durkheim (12 ans en $\left.5^{\mathrm{e}}\right)$. En fait, il décrit la bataille des Vosges. Il se penche sur un incident antisémite qui a suivi la défaite qu'il croise avec un texte de Durkheim qui écrivait se souvenir parfaitement de cette période (dans Dagan, Essai sur l'antisémitisme de 1896). L'auteur explique que la défaite a eu son poids sur la conscience politique de Durkheim, sa foi en la science et son pouvoir de régénération de la nation. Il a su trouver des citations pertinentes en rapport avec l'évocation de cette époque (dans un compte-rendu d'un ouvrage de Fouillée paru en 1907, dans l'article sur l'histoire de l'université de 1918). Il esquisse alors (en citant Bouglé) le portrait de cette "génération de jeunes hommes hantés par trois problèmes : le redressement national, l'émancipation laïque et l'organisation sociale et économique» (p. 252). Ce portrait de Durkheim en représentant d'une génération, concept intermédiaire entre l'individu et la société, est assez convaincant. Il serait à creuser, comme bien d'autres éléments du livre.

14 On le voit, cet ouvrage est riche de contributions d'historiens qui n'hésitent pas à chercher des informations inédites dans les archives des Vosges, dans les registres de recensements. On en retire un ensemble de faits sur le " contexte » spinalien et plus généralement vosgien, sur l'état de la "communauté juive" autour de 1870 . On pourrait évidemment souligner les petites et grandes frustrations qu'il occasionne par moments, on l'a déjà dit, quand ce contexte n'est pas suffisamment rapporté à l'individu Durkheim au centre de l'enquête. De quoi était-il porteur ou représentatif ? De quelle communauté était-il caractéristique ? De quoi et en quoi était-il fait, de quoi s'est-il détaché ensuite? Les questions restent ouvertes, en partie. Le mérite du livre aura été de les poser pour la première fois. 